\title{
DISCOPATIA CERVICAL E TORACOLOMBAR EM CÃES: REVISÃO
}

BORGES, Gabriela Augusta de Andrade Barbosa ${ }^{1}$

LÉGA, Elzylene ${ }^{2}$

\begin{tabular}{|c|c|c|}
\hline Recebido em: 2019.06 .25 & Aprovado em: 2020.03 .16 & ISSI \\
\hline
\end{tabular}

RESUMO: Existem duas categorias de discopatias, a cervical e a toracolombar. As lesões do disco cervical ocorrem mais comumente entre as vértebras cervicais C2-C3. As lesões do disco toracolombar ocorrem mais comumente entre as vértebras torácicas T12-T13 e toracolombares T13-L1. A hérnia de Hansen tipo I ocorre pela extrusão do disco intervertebral. A hérnia de Hansen tipo II ocorre pela protrusão do disco intervertebral. Na hérnia de Hansen tipo III ocorre lesão medular sem compressão. Na discopatia cervical o principal sinal clínico é a cervicalgia. Na discopatia toracolombar os sinais clínicos incluem variados graus de ataxia, paraparesia e paraplegia. O diagnóstico da doença do disco intervertebral (DDIV) é baseado na anamnese, exame físico, neurológico e complementares. A confirmação ocorre pelo exame complementar de imagem. Dentre as opções de exames complementares de imagem estão a radiografia simples (Exame de triagem), mielografia, tomografia, mielotomografia e, a melhor opção atualmente, a ressonância magnética. A sensibilidade à dor profunda é um importante indicador do prognóstico. $\mathrm{O}$ tratamento pode ser conservador, cujo principal aspecto é o confinamento estrito; ou por meio da descompressão cirúrgica. $\mathrm{Na}$ discopatia cervical os procedimentos cirúrgicos descompressivos incluem laminectomia dorsal, hemilaminectomia (Raramente indicadas) e slot ventral (Método de eleição). Na discopatia toracolombar os procedimentos cirúrgicos descompressivos incluem laminectomia dorsal (Funkquist tipo A e B, profunda e modificada), hemilaminectomia e mini-hemilaminectomia ou pediculectomia. No pós-operatório, muitos profissionais recomendam a fisioterapia e acupuntura para ajudar na recuperação.

Palavras-chave: Doença neurológica. Hansen. Hérnia de disco. DDIV.

\section{CERVICAL AND TORACOLOMBAR DISCOPATHY IN DOGS: REVIEW}

\begin{abstract}
SUMMARY: There are two categories of discopathies, the cervical and the thoracolumbar. Cervical disc lesions most commonly occur between the $\mathrm{C} 2-\mathrm{C} 3$ cervical vertebrae. The thoracolumbar disc lesions occur most commonly between the thoracic vertebrae T12-T13 and thoracolumbar vertebrae T13-L1. Hansen hernia type I occurs by extrusion of the intervertebral disc. The Hansen hernia type II occurs by the protrusion of the intervertebral disc. In the Hansen hernia type III, spinal cord injury occurs without compression. In cervical discopathy the main clinical sign is cervicalgia. In thoracolumbar discopathy clinical signs include varying degrees of ataxia, paraparesis and paraplegia. The diagnosis of intervertebral disc disease (DDIV) is based on anamnesis, physical, neurological and complementary examination. Confirmation occurs through complementary imaging. Among the options for complementary imaging tests are simple radiography (screening test), myelography, tomography, myelotomography and, currently the best option, magnetic resonance imaging. Sensitivity to deep pain is an important prognostic indicator. The treatment can be conservative, whose main aspect is the strict confinement; or by means of surgical decompression. In cervical discopathy, decompressive surgical procedures include dorsal laminectomy, hemilaminectomy (Rarely indicated) and ventral slot (Election method). In thoracolumbar discopathy the decompressive surgical procedures include dorsal laminectomy (Funkquist type A and B, deep and modified), hemilaminectomy and mini-hemilaminectomy or pediculectomy. In the postoperative period, many practitioners recommend physical therapy and acupuncture to assist in recovery.
\end{abstract}

Keywords: Neurological disease. Hansen. Herniated disc. DDIV.

\footnotetext{
${ }^{1}$ Médica Veterinária - FE/FAFRAM

${ }^{2}$ Dra.Coordenadora do Curso de Medicina Veterinária FE/FAFRAM
} 


\section{INTRODUÇÃO}

A coluna vertebral do cão é composta por 7 vértebras cervicais (C1-C7), 13 torácicas (T1T13), 7 lombares (L1-L7), 3 sacrais (S1-S3) e aproximadamente 20 coccígeas. A medula espinhal é dividida em quatro regiões funcionais: cervical cranial (C1-C5), intumescência cervicotorácica (C6-T2), intumescência toracolombar (T3-L3) e intumescência lombossacra (L4-S3). Alguns segmentos da medula espinhal encontram-se na vértebra correspondente, outros não. A localização das lesões neurológicas refere-se aos segmentos da medula espinhal, por isso é importante entender a relação entre as vértebras e os segmentos de medula espinhal (SHARP; WHEELER, 2005; PLATT; OLBY, 2012).

Os corpos intervertebrais são unidos através de discos intervertebrais, exceto as duas primeiras vértebras cervicais (Atlas e Axis) e as vértebras sacrais fusionadas (SHARP; WHEELER, 2005). Os discos intervertebrais (DIVs) são compostos por uma camada externa fibrosa, denominada anel fibroso, e uma porção central gelatinosa, denominada núcleo pulposo. Com o envelhecimento, o núcleo pulposo vai sendo gradualmente substituído por fibrocartilagem. A função dos DIVs é absorver o impacto sem prejudicar a flexibilidade da coluna vertebral (JANSSENS, 1991; TAYLOR, 2010).

A doença do disco intervertebral (DDIV) é uma das enfermidades mais comuns que acometem o sistema nervoso central (SNC), sendo a maior causa de paraplegia em cães, principalmente nos cães de pequeno porte. Existem basicamente duas grandes categorias de discopatias, a cervical e a toracolombar, sendo que a discopatia toracolombar é responsável por aproximadamente $85 \%$ dos casos e a cervical por 15\% dos casos (DA COSTA, 2001). A tetraparesia não ambulatória aparenta ser uma manifestação incomum na discopatia cervical (PADILHA FILHO; SELMI, 1999), ocorrendo em apenas 10\% dos casos (SEIM III, 2008b).

As lesões do disco cervical ocorrem mais comumente em C2-C3, com envolvimento decrescente progressivo a partir de C3-T1 (TOOMBS; WATERS, 2007). Em um estudo realizado por Padilha Filho e Selmi (1999), no qual atendeu-se 17 animais com discopatia cervical, constatou-se que os discos intervertebrais (DIVs) mais acometidos, em ordem decrescente, foram: C2-C3 (40\%), C3-C4 (25\%), C4-C5 (15\%), C5-C6 e C6-C7 (10\%).

As lesões do disco toracolombar ocorrem mais comumente entre T11-L2, com ênfase em T12-T13 e T13-L1. De L1-L6 há envolvimento decrescente em sentido caudal e a partir de T12 há envolvimento decrescente em sentido cranial (TOOMBS; WATERS, 2007). Isso se deve ao fato de que os DIVs entre T1-T11 são estabilizados pelos ligamentos intercapitais, tornando a protrusão ou extrusão nessas regiões menos provável (LE COUTEUR; GRANDY, 2008). 
Segundo Selmi (2015); Dewey e Da Costa (2017b), as lesões entre T12-T13 e T13-L1 acometem principalmente os cães de pequeno porte, contudo relatam que nos cães de grande porte os discos mais afetados são entre L1-L2 e L2-L3.

\section{ETIOPATOGENIA DA DISCOPATIA EM CÃES}

A doença do disco intervertebral (DDIV) foi classificada pela primeira vez por Hansen. A ruptura total do anel fibroso acarretará a extrusão do núcleo pulposo no canal vertebral, causando hérnia de Hansen tipo I. A ruptura parcial do anel fibroso acarretará a protrusão do núcleo pulposo, causando a hérnia de Hansen tipo II (DENNY; BUTTERWORTH, 2006; MAI, 2014).

A hérnia de Hansen tipo I tem maior incidência nas raças condrodistróficas (Dachshund, Poodle toy, Pequinês, Beagle, Lhasa apso, Shih tzu, Chihuahua, Cocker spaniel e cães mestiços) com maior incidência entre os 3 a 6 anos de idade (PADILHA FILHO; SELMI, 1999; TAYLOR, 2010). Entretanto, outros autores relatam incidência entre os 2 a 7 anos de idade, com pico em torno dos 4 a 5 anos (TOOMBS; WATERS, 2007; MAI, 2014). Os cães da raça Dachshunds apresentam risco dez a doze vezes maior de ter a lesão, quando comparados a cães de outras raças (SELMI, 2015). A hérnia de Hansen tipo I ainda pode ser diagnosticada, em menor frequência, em cães de médio a grande porte com maior faixa etária, principalmente nas raças Basset hound, Labrador retriever, Dálmata, Doberman e Pastor alemão. A hérnia de Hansen tipo II tem maior incidência em raças não condrodistróficas e de maior faixa etária, entre 6 a 8 anos de idade (TAYLOR, 2010). Segundo Toombs e Waters (2007), as alterações clinicamente significativas são observadas em cães com 8 a 10 anos de idade.

Recentemente tem-se descrito uma manifestação discal na qual se observa lesão medular sem compressão, que é denominada extrusão discal aguda não compressiva ou hérnia de Hansen tipo III (SELMI, 2015). Tal evento ocorre caso um disco com núcleo pulposo de hidratação normal seja colocado sob estresse intenso, o anel fibroso pode se romper e parte do núcleo pulposo gelatinoso normal pode explodir para o canal vertebral provocando uma contusão da medula espinhal. Uma vez que o material nuclear não apresenta alterações degenerativas, ele se difunde na gordura peridural, deixando apenas as lesões secundárias que podem ser atribuídas à contusão aguda da medula espinhal, com pouca ou nenhuma compressão do órgão. Tais hérnias são comumente associadas a traumas como acidentes automobilísticos ou impactos com obstáculos, mas também podem ser associadas a exercícios vigorosos (MAI, 2014).

As lesões traumáticas ao sistema nervoso desencadeiam rapidamente uma resposta inflamatória e uma série de alterações bioquímicas e metabólicas, que contribuem para a lesão secundária subsequente ao impacto primário. Esse processo inicia-se dentro de segundos após a 
lesão, embora grande parte da necrose celular ocorra nas primeiras 24 horas da lesão, a morte celular apoptótica pode ter continuidade durante semanas. As alterações patológicas induzidas pela compressão são: desmielinização, edema vasogênico, degeneração axonal e necrose neuronal. $\mathrm{O}$ edema vasogênico se deve, supostamente, a obstrução da drenagem venosa e às alterações na permeabilidade dos vasos sanguíneos. Em casos de compressão mais crônica, ocorre necrose e apoptose das células gliais, neurônios e axônios devido a isquemia (OLBY; JEFFERY, 2007).

Foram descritos dois tipos de alterações metaplásicas, a metaplasia condroide e a fibroide. Na metaplasia condroide ocorre desvio das concentrações dos glicosaminoglicanos, perdas no teor hídrico e proteoglicano e aumento no conteúdo de colágeno, portanto o DIV torna-se mais cartilaginoso perdendo progressivamente suas qualidades hidroelásticas de absorção de impacto. Frequentemente o núcleo degenerado sofre calcificação, comprometendo ainda mais seu funcionamento. A metaplasia fibroide deixa o DIV com níveis mais elevados de glicosaminoglicanos e conteúdo mais baixo de colágeno, em comparação aos DIV condrodistróficos. O núcleo dos DIVs não condrodistróficos conserva consistência mais semelhante a um gel, devido ao seu teor hídrico mais alto e à mineralização rara. Por isso, a metaplasia fibroide ocorre mais tardiamente em raças não condrodistróficas (TOOMBS; WATERS, 2007).

A extrusão do disco tipo I geralmente resulta em sinais clínicos mais graves que a protrusão do disco tipo II, embora a distorção mecânica e a compressão da medula espinhal causadas pela protrusão do tipo II possam ser maiores. A extrusão do núcleo pulposo é mais comum de forma aguda e subaguda em discos que estejam sofrendo degeneração condroide; enquanto a compressão progressiva ou lenta da medula espinhal acompanha frequentemente a protrusão de disco tipo II que esteja sofrendo degeneração fibroide (LE COUTEUR; GRANDY, 2008; WIDMER; THRALL, 2014).

\section{SINAIS CLÍNICOS DA DISCOPATIA EM CÃES}

Entre as variáveis que afetam os sinais clínicos da discopatia em cães estão: a localização da lesão, o volume de material extruído, o grau de compressão medular e a força/velocidade de extrusão. O início desses sinais pode ocorrer dias ou semanas antes que traumas ou movimentações causem maior extrusão (SEIM III, 2008c).

A cervicalgia é o achado clínico principal e mais comum em cães com discopatia cervical. Os cães acometidos geralmente mantêm a cabeça e o pescoço rígidos, choram quando movidos e 
podem exibir espasmos na musculatura cervical (LE COUTEUR; GRANDY, 2008). Na discopatia toracolombar os sinais clínicos são agudos e gradativos, começando com ataxia, avançando para paraparesia, paraplegia e finalmente, paraplegia com ausência de dor profunda (DA COSTA, 2001).

O neurônio motor inferior (NMI) localiza-se no núcleo cerebral e medula espinhal, enquanto o neurônio motor superior (NMS) localiza-se no córtex e tronco cerebral. O NMS inibe o NMI, pois, se o NMI não é inibido, os reflexos do nervo cranial ou espinhal tornam-se hiperativos. Portanto, um reflexo espinhal exagerado traduz uma lesão localizada acima deste nível, enquanto um reflexo espinhal deprimido ou ausente traduz uma lesão localizada no caminho do reflexo (CHRISMAN, 1985). Portando, lesões nos NMI causam hiporreflexia ou arreflexia, paresia ou paralisia flácida e atrofia muscular neurogênica, considerada rápida. Lesões nos NMS causam normorreflexia ou hiperreflexia, paralisia espástica e atrofia muscular por desuso, considerada lenta. A anamnese ajuda na distinção das atrofias (FERNÁNDEZ; BERNARDINI, 2010).

Lesões no segmento cervical cranial causam sinais clínicos relacionados aos NMS em ambos os membros; lesões no segmento da intumescência cervicotorácica causam sinais clínicos relacionados aos NMI nos membros torácicos (MT) e sinais clínicos relacionados aos NMS nos membros pélvicos (MP); lesões no segmento da intumescência toracolombar não causam sinais clínicos nos MT e nos MP, causando sinais clínicos relacionados aos NMS; lesões no segmento da intumescência lombossacra também não causam sinais clínicos nos MT e nos MP, causando sinais clínicos relacionados aos NMI. Contudo, em cães há um trato motor ascendente localizado dorsolateralmente na borda da substância cinzenta dos segmentos da medula espinhal lombar que inibe os músculos extensores dos MT. Uma lesão grave nessa via pode causar a síndrome de Schiff-Sherrington, presente nos MT em lesões graves na intumescência toracolombar (SHARP; WHEELER, 2005).

Distúrbios da micção relacionados ao NMI causam redução do tônus do esfíncter, portanto a urina tende a escoar continuamente; já os relacionados ao NMS causam aumento do tônus do esfíncter, retendo a urina que só é eliminada quando a pressão intravesical excede a pressão do esfíncter (SHARP; WHEELER, 2005; TAYLOR, 2010).

\section{DIAGNÓSTICO DA DISCOPATIA EM CÃES}

Diversas doenças podem acometer o SNC e podem ser classificadas de acordo com a causa, usando o mnemônico DAMNITV: D Degenerativo; A Anômalo; M Metabólico; N Neoplásico, nutricional; I Inflamatório, infeccioso e idiopático; $\mathrm{T}$ Traumático, tóxico e V 
Vascular. A natureza do início e evolução da doença é frequentemente útil para classificar os diagnósticos diferenciais prováveis para um paciente específico (PLATT; OLBY, 2012; DEWEY; DA COSTA, 2017a).

Os diagnósticos diferenciais mais prováveis para discopatia cervical incluem siringomielites, neoplasia, instabilidade atlantoaxial, discoespondilite, fratura, subluxação e espondilomielopatia cervical ou síndrome de Wobbler. Os diagnósticos diferenciais mais prováveis para discopatia toracolombar incluem fratura, luxação, discoespondilite, malformação congênita, neoplasia, osteomielite vertebral, embolia fibrocartilaginosa e síndrome da cauda equina (TOOMBS; WATERS, 2007; SEIM III, 2008bc).

Geralmente, pode-se estreitar os diagnósticos diferenciais por meio do uso apropriado de exame físico, hematológico, perfil bioquímico sérico, análise do líquor e exames radiográficos (SEIM III, 2008bc). A análise do líquor auxilia no diagnóstico como forma de exclusão de doenças infecciosas e inflamatórias da medula espinhal (ARIAS et al., 2007; LE COUTEUR; GRANDY, 2008; TAYLOR, 2010).

O diagnóstico da DDIV é baseado na anamnese, exame físico, neurológico e complementares, como hemograma e perfil bioquímico. A confirmação ocorre pelo exame complementar de imagem (SELMI, 2015).

\subsection{EXAME RADIOGRÁFICO SIMPLES}

Os achados radiográficos indicativos de herniação de disco intervertebral incluem estreitamento do espaço discoide e das articulações facetarias, forame intervertebral pequeno e/ou enevoado e presença de material calcificado no canal espinhal (JEFFERY, 1995; SEIM III, 2008bc; BRISSON, 2010). No entanto, a diminuição dos espaços intervertebrais nem sempre é indicativa de DDIV e dever ser interpretada no contexto dos sinais clínicos. Os espaços intervertebrais T10-T11 e L5-L6 normalmente são mais estreitos que os demais. A radiografia simples não deve ser utilizada para determinar o local e a gravidade da lesão antes de uma descompressão cirúrgica, mas sim para excluir outras condições como fratura, luxação, discoespondilite e neoplasias resultando em lise óssea (WIDMER; THRALL, 2014; DE LAHUNTA; GLASS; KENT, 2015a). 


\subsection{MIELOGRAFIA}

A mielografia identifica o local da lesão e o grau de compressão, mas não informa a condição da medula espinhal, entretanto, ainda é útil quando a tomografia computadorizada (TC) ou ressonância magnética $(\mathrm{RM})$ não estão disponíveis (WIDMER; THRALL, 2014). É considerada um meio de diagnóstico bastante acurado para a doença do disco intervertebral em cães, inclusive determinando o lado correto em 89 a 100\% dos casos (KERWIN; LEVINE; HICKS, 2012).

A alteração mais observada na mielografia é o desvio focal da coluna de contraste, em direção dorsal ou dorsolateral, podendo haver lateralização do desvio na projeção ventrodorsal, como indicado nas figuras 7 e 8 (MACIAS et al., 2002). Em um estudo com 60 cães, a mielografia mostrou a presença de uma massa extradural em 57 deles, sendo que 24 apresentaram lateralização clínica ou mielográfica dos sinais (MCKEE, 1992).

A incidência de crises convulsivas pós mielográficas com injeção de iohexol varia de $0 \%$ a $10 \%$ e tem sido associada ao peso do animal (maior peso), volume de contraste injetado (maior volume), injeção cerebelomedular, localização da lesão (região cervical mais susceptível), sexo e raça (cães machos da raça Doberman mais susceptíveis) (BRISSON, 2010).

\subsection{TOMOGRAFIA COMPUTADORIZADA (TC)}

A TC é excelente na identificação, localização, lateralidade e extensão da compressão medular em cães condrodistróficos acometidos pela hérnia de Hansen tipo I mineralizada. Porém a mielotomografia é frequentemente útil quando não se identifica uma lesão nesses animais devido ao edema medular. Além disso, a TC é rápida, não tem efeitos colaterais e pode ser formatada em plano tridimensional para melhores fins diagnósticos. A mielotomografia pode ser realizada por injeção de meio de contraste iodado no espaço subaracnoide, geralmente utilizando $25 \%$ da dose mielográfica regular. Quando a TC é realizada após a mielografia, o saco tecal fica hiperatenuante, permitindo a diferenciação mais precisa entre doenças medulares intradurais e extradurais (BRISSON, 2010; MAI, 2014).

Em um estudo, Shimizu et al. (2009) examinaram 11 cães da raça Dachshund através da tomografia computadorizada, antes e depois da injeção do contraste pré-aquecido (Iohexol) na dose de $0,45 \mathrm{ml} / \mathrm{kg}$ no espaço subaracnoide através do forame L5-L6 e os resultados foram comparados. Em alguns casos, o exame pós-contraste identificou a lesão compressiva, que não foi identificada no exame pré-contraste. Portanto, na experiência clínica dos autores, o realce do contraste foi necessário para diagnosticar a DDIV em cães usando TC. 


\subsection{RESSONÂNCIA MAGNÉTICA (RM)}

A RM é considerada o melhor método para diagnóstico precoce de degeneração de disco e para visualização da medula espinhal, DIVs e estruturas associadas. Geralmente, a RM é uma ferramenta útil para orientar a decisão cirúrgica em relação ao tamanho e localização da laminectomia toracolombar, porém a imagem da RM tende a subestimar o tamanho do material extrusado sendo indicado a realização de uma janela cirúrgica um pouco maior (BRISSON, 2010).

A RM permite a identificação da degeneração do disco e diferenciação entre hérnia de Hansen tipo I ou II, detectando ainda alterações estruturais no interior da medula espinhal e espaço epidural, como hemorragia e inflamação associadas às extrusões de disco (KERWIN; LEVINE; HICKS, 2012; MAI, 2014).

\subsection{EXAME NEUROLÓGICO}

Frequentemente há a presença de afecções ortopédicas, como a displasia coxofemoral e a ruptura do ligamento cruzado cranial, concomitantemente a distúrbios neurológicos relacionados com a hérnia de Hansen tipo II, principalmente em cães idosos de porte grande. Por isso, também é preciso realizar um exame do aparelho locomotor. Entretanto uma doença músculo esquelética não causa ataxia proprioceptiva, por mais grave que seja (JEFFERY, 1995; FERNÁNDEZ; BERNARDINI, 2010).

O exame neurológico é uma extensão do exame físico geral e deve ser realizado após a anamnese e o exame físico (SEIM III, 2008c). É uma ferramenta importante no diagnóstico, pois permite definir a localização e a gravidade da lesão. Não devem ser administrados analgésicos antes da realização do exame neurológico, pois estes podem alterar o resultado. $\mathrm{O}$ exame neurológico precisa ser realizado com cuidado para evitar piora das lesões devido a movimentação excessiva (JEFFERY, 2001a; LE COUTER; GRANDY, 2008).

No exame neurológico, primeiramente avalia-se o estado mental, postura e marcha através da observação. Depois avalia-se o tônus muscular, reações posturais, reflexos espinhais, nervos cranianos e sensibilidade. A utilização de uma ficha de exame neurológico é uma ferramenta auxiliar importante na realização do exame (LORENZ; KORNEGAY, 2004; FERNÁNDEZ; BERNARDINI, 2010).

As alterações do estado mental incluem depressão, esturpor, coma e demência. As alterações da atitude incluem principalmente head tilt e head turn e da postura incluem rigidez 
por descerebelação, postura de Schiff-Sherrington, cifose, lordose, escoliose, postura plantígrada e palmígrada. As alterações da marcha incluem claudicação, ataxia, paresia, plegia, tremor, miotonia e mioclonia. Para a avaliação das reações posturais pode-se realizar o teste do posicionamento proprioceptivo, do saltitamento, da resposta de posicionamento não visual e visual. Frequentemente os dois primeiros testes são suficientes. Caso as respostas posturais forem ambíguas ou duvidosas, pode-se ainda realizar o teste da hemideambulação, carrinho de mão e impulso postural extensor (DEWEY; COSTA; THOMAS, 2017).

A parte de maior importância no exame neurológico para a localização da lesão e atribuição desta a uma das quatro regiões funcionais da medula espinhal é a avaliação dos reflexos espinhais, pois nos permite determinar quais os motoneurônios afetados (PLATT; OLBY, 2012). Entretanto, quando as lesões medulares são múltiplas, o exame neurológico pode não identificar uma segunda lesão cranial à primeira (JEFFERY, 2001b).

Os reflexos espinhais mais confiáveis e que devem ser sempre realizados incluem avaliação do tônus muscular, reflexo patelar, flexor, perineal e cutâneo do tronco. Porém, é válido lembrar que existem ainda os reflexos miotáticos gastrocnêmico, tibial cranial, bicipital, tricipital e extensor radial do carpo. O tônus muscular é o indicador mais confiável de lesão do NMS que o reflexo patelar. O reflexo patelar avalia a integridade do nervo femoral e dos segmentos L4-L6, sendo o reflexo miotático mais confiável. A perda deste reflexo uni ou bilateral em cães com 10 anos ou mais é um fenômeno específico relacionado com a idade. O reflexo flexor avalia a integridade dos segmentos C6-T2 para o MT e L6-S2 para o MP. O reflexo perineal avalia a integridade dos segmentos S1-S3 e caudais, bem como dos nervos perineal e retal caudal. O reflexo cutâneo do tronco fica ausente na região toracolombar cerca de dois corpos vertebrais caudais a lesão. Nas regiões cervical e sacral não existe esse reflexo (DEWEY; COSTA; THOMAS, 2017).

Para a realização de um exame neurológico completo, segue a avaliação dos nervos cranianos $(\mathrm{NC})$. O teste de resposta à ameaça e o teste do movimento de acompanhamento avaliam o nervo óptico (NC II) e nervo facial (NC VII). O primeiro teste resulta no ato de piscar o olho e certo recuo da cabeça. No segundo teste, o animal costuma acompanhar o movimento das mãos do clínico com os olhos. O teste de reflexo pupilar à luz avalia os nervos óptico (NC II) e oculomotor (NC III). Na luminosidade deve ocorrer miose e na meia-luz deve ocorrer midríase. A pupila contralateral também deve ser responsiva, tal evento é denominado reflexo pupilar indireto ou consensual. O teste do reflexo oculocefálico avalia os nervos oculomotor (NC III), troclear (NC IV), abducente (NC VI) e vestibulococlear (NC VIII). Neste teste o animal deve apresentar movimentos simétricos do globo ocular e nistagmo horizontal e vertical de acordo com a direção do movimento. O reflexo da sensibilidade nasal, avalia o nervo trigêmeo (NC V). Deve 
ser feito de maneira delicada e as cegas, a resposta normal corresponde ao afastamento abrupto da cabeça. Os reflexos corneano e palpebral avaliam os nervos trigêmeo (NC V) e facial (NC VII). O primeiro teste consiste na estimulação da córnea e o segundo teste consiste na estimulação das margens palpebrais. Ambos os testes induzem ao reflexo ipsilateral de piscar. O primeiro teste ainda pode induzir a retração da órbita, avaliando também o nervo abducente (NC VI) (BRAUND; SHARP, 2007).

Em relação ao teste de resposta à ameaça, deve-se testar em cada olho individualmente, com o olho contralateral coberto e tomar cuidado para não tocar em nenhum dos pelos faciais ou criar muita turbulência de ar, pois os reflexos palpebrais e corneanos serão ativados. No cão, observa-se uma perda unilateral da resposta à ameaça em pacientes com uma grande lesão cerebelar unilateral do mesmo lado que o deficit de ameaça. Já em relação ao reflexo pupilar à luz, deve-se lembrar que a resposta direta é maior que a indireta ou consensual (JEFFERY, 2001a; DE LAHUNTA; GLASS; KENT, 2015b).

Para a avaliação sensorial segue-se a palpação de cada vértebra da coluna, dos membros, flexão da cauda e cabeça, além do estímulo a dor superficial e profunda. A dor superficial é aguda e origina-se comumente na pele, enquanto a dor profunda é sentida como queimação e se origina na pele ou em estruturas mais profundas. Para avaliar a dor profunda, usa-se uma pinça hemostática para pinçar os dígitos e produzir dor no periósteo, se necessário pode-se pinçar a cauda. A resposta deve ser comportamental, por exemplo a vocalização, a retirada do membro não é suficiente. Se a dor superficial estiver presente, não é necessário produzir dor profunda no animal (SEIM III, 2008a; DE LAHUNTA; GLASS; KENT, 2015a; DEWEY; DA COSTA; THOMAS, 2017).

A sequência da deterioração correlaciona-se com o diâmetro das fibras. Com a compressão da medula espinhal, grandes fibras perdem a função antes que pequenas fibras sejam afetadas (LORENZ; KORNEGAY, 2004). Portanto, primeiro se perde a propriocepção, em seguida se perde a atividade motora voluntária, a sensação da dor superficial e da dor profunda; respectivamente. A reabilitação clínica é em disposição contrária (DA COSTA, 2001; SEIM III, 2008a).

\section{PROGNÓSTICO}

Durante o exame físico é extremamente importante avaliar o reflexo de dor profunda, sendo este um importante indicador do prognóstico. Se estiver presente o prognóstico é favorável, 
se estiver ausente por mais de 48 horas o prognóstico é reservado a desfavorável e a probabilidade de recuperação é baixa (LORENZ; KORNEGAY, 2004; SEIM III, 2008a).

Contudo, um estudo realizado por Arias et al. (2007), em 8 cães paraplégicos e com ausência de dor profunda verificou-se melhora no quadro clínico e total recuperação em 62,5\% dos casos, além disso, em 4 cães tetraplégicos a descompressão cirúrgica foi efetiva. Os métodos descompressivos avaliados pelos autores foram slot ventral na região cervical e hemilaminectomia na região toracolombar.

\section{TRATAMENTO}

\subsection{Tratamento conservador}

O tratamento conservador é indicado em casos de dor, paresia leve, perda crônica da dor profunda, quando o tutor recusa a cirurgia ou não possui condições financeiras e presença de afecção sistêmica grave que contraindiquem a realização da cirurgia (BRAUND, 1996; TOOMBS; WATERS, 2007).

O aspecto essencial do manejo conservador bem-sucedido da DDIV é o confinamento estrito em gaiola, aplicado por 4 a 6 semanas e pode incluir baixas doses anti-inflamatórias de corticosteroides e relaxantes musculares. Este repouso forçado auxilia na resolução da inflamação medular e intra-discal, facilitando a estabilização do disco por meio de fibrose. A seguir, recomenda-se 3 a 4 semanas de retorno gradual a atividade. No entanto, a terapia clínica sozinha é frequentemente ineficaz para o tratamento da discopatia cervical, uma razão para a falta de resposta ao repouso restrito é a dificuldade de manter a imobilidade total da coluna cervical. Russell e Griffiths (1968) relataram 36\% de recorrência dos sinais clínicos em um grupo de 110 cães gerenciados pelo método conservador. Se a terapia clínica não surtir efeito entre 7 a 10 dias, o paciente deve ser avaliado novamente e a descompressão cirúrgica considerada (COATES, 2000; SEIM III, 2008bc; LE COUTEUR; GRANDY, 2008).

Os cuidados fundamentais de enfermagem incluem monitorização do consumo de água e alimento, da micção e defecação, banhos e troca da cama regulares para evitar assaduras e úlceras de decúbito, além de fisioterapia para manutenção da massa muscular e amplitude dos movimentos articulares. A retenção urinária é usual, havendo a necessidade de esvaziamento da bexiga através de compressão manual ou cateterização uretral, três a quatro vezes por dia (TOOMBS; WATERS, 2007).

Os corticosteroides utilizados em dose anti-inflamatória reduzem o edema vasogênico diminuindo o efeito compressivo, reduz a inflamação das raízes nervosas e isquemia devido ao 
efeito anti-prostaglandina, aliviando assim a dor (JEFFERY, 1995; BAHR ARIAS; SEVERO; TUDURY, 2007). A utilização de prednisona na dose de $0,5 \mathrm{mg} / \mathrm{kg}$, a cada 24 horas, durante 15 dias, em seguida a cada 48 horas, durante mais 15 dias é recomendada nesses casos (DA COSTA, 2001).

\subsection{Tratamento cirúrgico}

A descompressão cirúrgica é indicada para pacientes com deficits neurológicos graves ou progressivos, mas também tem sido recomendada para pacientes com deficits neurológicos leves (BRISSON, 2010).

Os procedimentos neurocirúrgicos para o tratamento de discopatia cervical no cão incluem fenestração, laminectomia dorsal, hemilaminectomia e slot ventral. A técnica mais recomendada é o slot ventral, devido a melhor visualização dos discos ventrais e ventrolaterais, manipulação mínima da medula espinhal, menor tempo de operação e recuperação rápida (SEIM III; WITHROW, 1996). Os procedimentos cirúrgicos descompressivos para o tratamento da discopatia toracolombar no cão incluem laminectomia dorsal (Funkquist tipo A e B, modificada e profunda), hemilaminectomia e mini-hemilaminectomia ou pediculectomia (SEIM III, 2008c).

Devido à ausência de dados clínicos objetivos sobre o uso de neuroprotetores na medicina veterinária, aplicações clínicas comprovadas na medicina humana ou modelos experimentais são adotadas. Alguns médicos veterinários defendem uma dose única de succinato sódico de metilpredinisolona antes da cirurgia para reduzir quaisquer efeitos secundários. O emprego de altas doses de dexametasona é disseminado, porém sua eficácia não é comprovada (OLBY; JEFFERY, 2007).

A descompressão cirúrgica comparada ao tratamento conservador, apresenta taxa de recuperação mais rápida e menor possibilidade de persistência de deficits neurológicos. Porém os melhores resultados cirúrgicos são obtidos quando a descompressão é realizada em até 48 horas após o aparecimento dos sinais clínicos (TAYLOR, 2010). Em estudo realizado por Crawford e De Decker (2017), foram analisados 84 animais com DDIV, sendo que 53 foram tratados cirurgicamente e 31 de forma conservadora. O tratamento cirúrgico obteve taxa de 71,1\% (43 cães) de sucesso ou estabilização do quadro, em comparação ao tratamento conservador, que obteve taxa de 29,6\% (18 cães). Quanto a recorrência, a terapia cirúrgica apresentou taxa de 25,6\% (11 cães), em comparação a terapia conservadora, que apresentou taxa de 55,6\% (10 cães). 


\subsubsection{Técnicas Cirúrgicas Descompressivas}

O Slot ventral refere-se à criação de uma fenda na face ventral do espaço intervertebral entre as vértebras cervicais, para acessar e visualizar o canal espinhal ventral. A dimensão do slot ventral não deve exceder $50 \%$ de largura e $33 \%$ de comprimento do corpo vertebral. A remoção excessiva pode promover instabilidade e subluxações vertebrais (FIGUEIREDO; TUDURY; BAHR ARIAS, 2009). Esta técnica é indicada quando a lesão compressiva estiver localizada na porção ventral do canal vertebral (SEIM III 2008b).

Segundo um estudo realizado por Bahr Arias et al. (2007), avaliaram-se ainda os resultados clínicos após a descompressão cirúrgica em 45 cães com discopatia cervical (Slot cervical) e toracolombar (Hemilaminectomia), sendo que 35 cães $(77,8 \%)$ obtiveram recuperação total, $8(17,8 \%)$ parcial e $2(4,4 \%)$ não apresentaram alteração do quadro inicial, concluindo que os métodos descompressivos citados acima foram efetivos na melhora do quadro funcional.

A laminectomia refere-se à remoção da espinha e lâminas dorsais, além de quantidades variáveis das facetas articulares e pedículos em duas vértebras consecutivas (FIGUEIREDO; TUDURY; BAHR ARIAS, 2009). A técnica de Funkquist A refere-se à remoção da lamina dorsal, facetas articulares e pedículo até o nível médio da medula espinhal enquanto a técnica de Funkquist B refere-se à remoção da lâmina dorsal preservando as facetas articulares e pedículos. A técnica dorsal modificada é semelhante à técnica de Funkquist $\mathrm{B}$, refere-se à remoção caudal das facetas articulares inteiras e ventral das lâminas, causando exposição adicional do canal vertebral. A técnica dorsal profunda refere-se à remoção da lâmina dorsal, facetas articulares e pedículo até o nível ventral da medula espinhal (SEIM III 2008c).

A técnica de Funkquist A proporciona melhor visualização do canal vertebral lateral em comparação a laminectomia dorsal modificada e técnica de Funkquist B, sendo indicada quando as lesões compressivas estão localizadas no canal vertebral dorsal, lateral ou ventrolateral. A técnica de Funkquist B é indicada quando as lesões compressivas estão localizadas no canal vertebral dorsal. A laminectomia dorsal modificada é indicada quando as lesões compressivas estão localizadas no canal vertebral dorsal e ventrolateral. A laminectomia dorsal profunda proporciona excelente visualização do canal vertebral dorsal, lateral e ventral sendo indicada em casos de descompressão máxima (SEIM III, 2008c).

A hemilaminectomia refere-se à remoção unilateral das lâminas e pedículos dorsolaterais, além das facetas articulares. A hemilaminectomia é indicada quando as lesões compressivas estão localizadas no canal vertebral lateral, dorsolateral e ventrolateral, sendo preferível à laminectomia dorsal por preservar melhor a integridade da coluna, ser menos traumática, reduzir as chances de cicatrizes causarem compressão medular e pode ser realizada unilateralmente em até três 
vertebras consecutivas sem causar instabilidade vertebral. Para alcançar o canal vertebral e visualizar a medula espinhal, tanto na laminectomia, quando na hemilaminectomia, as camadas ósseas podem ser removidas com broca pneumática ou elétrica. O material discal extrusado é removido cuidadosamente do canal vertebral com a ajuda de pinças mosquito, pequena cureta, removedores de cálculo dentário, esculpidores ou espátulas para resina (SEIM III, 2008c; FIGUEIREDO; TUDURY; BAHR ARIAS, 2009).

Em um estudo realizado por McKee (1992), a hemilaminectomia foi efetiva em 93\% dos cães com discopatia toracolombar, em comparação a laminectomia dorsal, a qual foi efetiva em apenas $40 \%$ dos cães com discopatia toracolombar submetidos à essa técnica.

\subsubsection{Fenestracão}

A fenestração é a criação de uma janela, ou fenestra, no anel fibroso para remover o núcleo pulposo a partir de qualquer espaço intervertebral. Pode ser realizada por via dorsal, dorsolateral, lateral ou ventral. Esta técnica tem sido recomendada como forma profilática para reduzir a recidiva de extrusão em outro espaço de disco (FIGUEIREDO; TUDURY; BAHR ARIAS, 2009).

Os discos intervertebrais protuídos entre C2-C3 até C6-C7 são habitualmente submetidos a fenestração profilática (TOOMBS; WATERS, 2007). Segundo um estudo realizado por Padilha Filho e Selmi (1999), concluiu-se que a fenestração ventral gera resultados satisfatórios com índice de recidiva nulo, o que sugere uma boa alternativa no tratamento da discopatia cervical. Contudo, a fenestração não é efetiva se a tetraplegia resultar de extrusão discal (BOJRAB; CONSTANTINESCU, 1996). Além disso, é rara a extrusão do disco cervical num espaço intervertebral alternado, tornando desnecessária a fenestração ventral após um slot ventral. Como complicação, pode ocorrer tetraparesia por fenestração ultra agressiva (SEIM III, 2008b).

Segundo Taylor (2010), além da descompressão cirúrgica, muitos profissionais recomendam a fenestração concomitante de sítios adjacentes de alto risco (T11 a L3), para ajudar a diminuir a probabilidade de subsequentes herniações. Contudo, Seim III (2008c) afirma que a eficácia da fenestração profilática é variável e parece ser dependente da técnica cirúrgica descompressiva.

Segundo um estudo realizado por Tudury; Severo e Maciel (2004), a fenestração ventral e lateral dos DIVs cervicais e toracolombares, realizando-se fendas dorsais no anel fibroso de cadáveres caninos, pode causar extrusão do núcleo pulposo para o canal vertebral, causando consequências graves como a mielomalácia ascendente. 


\subsubsection{Outras técnicas}

A corpectomia consiste na criação de um slot ou fenda lateral entre dois corpos vertebrais adjacentes permititndo a remoção relativamente fácil do material discal sem provocar lesão iatrogênica à medula espinhal por excesso de manipulação. A corpectomia é uma alternativa à laminectomia dorsal no tratamento da discopatia toracolombar crônica ventral e ventrolateral (MOISSONNIER; MEHEUST; CARROZO, 2004).

Em um estudo com 107 cães, concluiu-se que a corpectomia foi efetiva em 91,4\% dos casos, com cães capazes de andar sem auxílio. A taxa de recorrência, no mesmo local, foi de 7,4\%. As complicações descritas foram, hemorragia e danos à raiz nervosa, respectivamente, em $24,3 \%$ e 5,6\% dos casos. Contudo, é uma opção cirúrgica viável para o tratamento de discopatia toracolombar crônica ventral (FERRAND et al., 2015).

Quando, durante a laminectomia dorsal, observa-se que as raízes nervosas de L7-S1 estão comprimidas dentro dos forames ou por facetas articulares aumentadas de tamanho, indica-se a realização da foraminotomia e facetectomia para estabelecer a descompressão completa. A facetectomia, principalmente bilateral, pode causar desestabilização da articulação lombossacral e se possível deve ser evitada (FIGUEIREDO; TUDURY; BAHR ARIAS, 2009).

O foraminotomia é um procedimento eficaz quando usado para tratamento da estenose lombossacral degenerativa (DLSS) e estenose foraminal isoladamente, ou juntamente com laminectomia dorsal. A DLSS envolve instabilidade do disco intervertebral L7-S1, compressão e inflamação da cauda equina e dos nervos L7 (GOMES; LOWRIE; TARGETT, 2018).

A durotomia é a incisão da dura-máter, praticada depois de uma laminectomia ou hemilaminectomia para descomprimir o edema da medula espinhal ou avaliar lesões intradurais. Não é útil para descompressão quando realizada após 2 horas do traumatismo, sendo de valor para determinar o prognóstico quando há suspeita de mielomalácia (FIGUEIREDO; TUDURY; BAHR ARIAS, 2009).

\section{PÓS-OPERATÓRIO}

Os animais devem ser monitorados para o controle de crises convulsivas nas primeiras 24 horas após a cirurgia, principalmente aqueles que foram submetidos a mielografia pré-operatória. Analgésicos devem ser fornecidos conforme a necessidade e geralmente a administração de corticosteroide é interrompida no pós-operatório (SEIM III, 2008bc). Os cuidados pós- 
operatórios de enfermagem são basicamente os mesmos citados para o tratamento conservativo (TOOMBS; WATERS, 2007).

\section{TERAPIAS COMPLEMENTARES}

\subsection{Fisioterapia}

Na medicina humana a fisioterapia existe há séculos. No entanto, a fisioterapia veterinária é uma disciplina muito mais jovem, que vem crescendo, e sua contribuição para a veterinária tem sido cada vez mais reconhecida (FENTEM JONES, 2012).

As modalidades fisioterápicas utilizadas após a descompressão medular são divididas em três categorias: 1) a terapia manual inclui a massoterapia, alongamentos, movimentação passiva e acupressão; 2) a termoterapia ou eletroterapia inclui a terapia pelo calor e frio, estimulação elétrica, laser e ultrassom terapêutico; 3) a terapia de exercício inclui exercícios de fortalecimento, flexibilidade, resistência, equilíbrio e propriocepção, além da hidroterapia. Algumas delas podem ser realizadas por enfermeiros e médicos veterinários, outras devem ser realizadas por fisioterapeutas veterinários especializados (SHARP, 2008; KLINE, 2017).

As técnicas fisioterápicas podem fazer uma grande diferença na recuperação neurológica, ortopédica e músculo-esquelética daqueles pacientes que passaram por trauma cirúrgico; complementando o trabalho do cirurgião veterinário e melhorando a qualidade de vida do paciente. Também pode ser usada no tratamento da dor e cicatrização de feridas. O papel do fisioterapeuta é garantir que o tratamento posterior desses pacientes seja tão bom e progressivo quanto o tratamento inicial. $\mathrm{O}$ feedback recebido inclui o consenso de que os pacientes que foram tratados com fisioterapia recebem alta mais cedo e com melhor funcionalidade em comparação a aqueles que não foram tratados (FENTEM JONES, 2012).

\subsection{ACupuntura}

A acupuntura é a estimulação de pontos específicos na superfície do corpo através da inserção de uma agulha, resultando em um efeito terapêutico ou homeostático. Esses pontos estão localizados em áreas com alta densidade de mastócitos, tecidos linfáticos e plexos arteriovenosos, além de regiões de inervação concentrada. A estimulação de diferentes pontos de acupuntura produz endorfinas o que gera analgesia a longo prazo, regula fatores pró-inflamatórios, reduz os danos dos radicais livres e melhora a microcirculação através de mecanismos neurais, neuro- 
humorais, neuromusculares e musculoesqueléticos. A estimulação dos acupontos pode ser alcançada através das técnicas: agulha seca, eletroacupuntura, aqua-acupuntura e moxabustão. A agulha seca é a técnica mais comumente usada em acupuntura veterinária. A acupuntura tem sido usada efetivamente para o tratamento de distúrbios neurológicos, como a DDIV, pois demonstrou acelerar a recuperação da função motora e promover analgesia. Embora os autores ainda recomendem cirurgias descompressivas, esses resultados justificam a acupuntura como tratamento adjuvante ao tratamento convencional (ROYNARD; FRANK; FOWLER, 2018).

\section{CONSIDERAÇÕES FINAIS}

As injúrias nervosas, como a DDIV, são relevantes na rotina clínica veterinária devido à alta frequência e causam, além da dor, sintomas graves como ataxia, paresia e plegia; que são sinais neurológicos que afetam negativamente a qualidade de vida dos animais.

Os diagnósticos diferenciais que mimetizam os sinais clínicos da DDIV são vários, portanto é de suma importância a realização de anamnese e exame neurológico adequados. O diagnóstico é confirmado por meio de exames de imagem, entre os quais a RM se destaca. Estudos comprovam que a mielotomografia possui maior acurácia que a TC padrão, porém na falta desses métodos de imagem avançados, a mielografia cumpre satisfatoriamente o seu papel.

O tratamento pode ser conservativo ou cirúrgico, entretanto, a literatura relata que a descompressão cirúrgica comparada ao tratamento conservador apresenta taxa de recuperação mais rápida e menor possibilidade de persistência de deficits neurológicos. As opções de técnicas cirúrgicas descompressivas descritas são diversas. De acordo com as literaturas pesquisadas e incluídas neste estudo, o tratamento de eleição para a discopatia cervical é o slot ventral; já para a discopatia toracolombar a hemilaminectomia é o tratamento de eleição, pois mostrou-se mais eficaz e menos invasiva em comparação a laminectomia dorsal. A fenestração é um método profilático e não descompressivo, cuja a eficácia divide opiniões.

As terapias alternativas como fisioterapia e acupuntura, têm ganhado espaço na área da medicina veterinária e mostrado bons resultados no tratamento adjuvante às terapias convencionais e na recuperação pós-operatória.

\section{REFERÊNCIAS}

BAHR ARIAS, M. V.; NISHIOKA, C. M.; GARCIA, C. O.; REIA, A. Z.; BARAÚNA JÚNIOR, D.; MARCASSO, R. A. Avaliação dos Resultados Clínicos Após Cirurgia Descompressiva em Cães com Doença de Disco Intervertebral. Arquivo Brasileiro de Medicina Veterinária e Zootecnia, Belo Horizonte, v. 59, n. 6, p. 1445-1450, 2007. 
BAHR ARIAS, M. V.; SEVERO, M. S.; TUDURY, E. A. Trauma Medular em Cães e Gatos: Revisão da Fisiopatogenia e do Tratamento Médico. Semina: Ciências Agrárias, Londrina, v. 28, n. 1, p. 115-134, 2007.

BOJRAB M. J.; CONSTANTINESCU G. M. Fenestração Discal Cervical. In: BOJRAB, M. J. Técnicas atuais em cirurgia de pequenos animais. 3 ed. São Paulo: Roca, 1996. p. 556-558.

BRAUND, K. G. Moléstia do Disco Intervertebral. In: BOJRAB, J. M. Mecanismos da Moléstia na Cirurgia dos Pequenos Animais. 2 ed. São Paulo: Manole, 1996. p. 1104-1116.

BRAUND, K. G.; SHARP, N. J. H. Exame e Localização Neurológicos. In: SLATTER, D. Manual de Cirurgia de Pequenos Animais, v. 1.3 ed. Barueri: Manole, 2007. p. 1092-1108.

BRISSON, B. A. Intervertebral Disc Disease in Dog. Veterinary Clinics of North America Small Animal Practice. Philadelphia, v. 40, n. 5, p. 829-858, 2010.

CHRISMAN, C. et al. Neurologia para o Clínico de Pequenos Animais. São Paulo: Roca, 1985. $336 \mathrm{p}$.

COATES, J. R. Intervertebral Disk Disease. Veterinary Clinics of North America Small Animal Practice, Philadelphia, v. 30, n. 1, p. 77-93, 2000.

CRAWFORD, A. H.; DE DECKER S. Clinical Presentation and Outcome of Dogs Treated Medically or Surgically for Thoracolumbar Intervertebral Disc Protrusion, Veterinary Record, v. 180, n. 23, p. 1-6, 2017.

DA COSTA, R.C. Disco Intervertebral: Bases para o Diagnóstico e Tratamento da Doença. Nosso Clínico, São Paulo, v. 20, n. 4, p. 18-26, 2001.

DE LAHUNTA, A.; GLASS, E.; KENT, M. Small Animal Spinal Cord Disease. In: Veterinary Neuroanatomy and Clinical Neurology. 4 ed. St. Louis: Saunders Elsevier, 2015a. p. 257-303.

DE LAHUNTA, A.; GLASS, E.; KENT, M. Visual Sistem. In: Veterinary Neuroanatomy and Clinical Neurology. 4 ed. St. Louis: Saunders Elsevier, 2015b. p. 409-454,

DENNY, R. H; BUTTERWORTH, J. S. Cirurgia Ortopédica em Cães e Gatos. 4 ed. São Paulo: Roca, 2006. p. 193-205.

DEWEY C. W.; DA COSTA R. C. Identificação e Histórico: as Primeiras Considerações. In: DEWEY C. W.; DA COSTA R. C. Neurologia Canina e Felina: Guia Prático. São Paulo: Guará, 2017a. p. 12-18.

DEWEY C. W.; DA COSTA R. C. Mielopatias: Doenças da Medula Espinhal. In: DEWEY C. W.; DA COSTA R. C. Neurologia Canina e Felina: Guia Prático. São Paulo: Guará, 2017b. p. 379-462.

DEWEY C. W.; DA COSTA R. C.; THOMAS W. B. Realizando o Exame Neurológico. In: DEWEY C. W.; DA COSTA R. C. Neurologia Canina e Felina: Guia Prático. São Paulo: Guará, 2017. p. 19-41. 
FENTEM JONES, H. Why Physiotherapy? Veterinary Record, v. 170, n. 8, p. 1-2, 2012.

FERNÁNDEZ, V. L.; BERNARDINI, M. Neurologia em cães e gatos. São Paulo: MedVet, 2010. $446 \mathrm{p}$.

FERRAND, F. X.; MOISSONNIER, P.; FILLEUR, A.; CACHON, T.; FAU, D.; VIGUIER, E.; CAROZZO, C. Thoracolumbar Partial Lateral Corpectomy for the Treatment of Chronic Intervertebral Disc Disease in 107 Dogs, Irish Veterinary Journal, v. 68, n. 27, p. 1-7, 2015.

FIGUEIREDO, M. L.; TUDURY E. A.; BAHR ARIAS M. V. Material, Instrumental e Procedimentos Básicos Utilizados na Cirurgia Espinhal de Cães e Gatos. In: TUDURY E. A.; POTIER G. M. A. Tratado de Técnica Cirúrgica Veterinária. São Paulo: MedVet, 2009. p. 323-340.

GOMES S. A.; LOWRIE M.; TARGETT M. Long-term Outcome Following Lateral Foraminotomy as Treatment for Canine Degenerative Lumbosacral Stenosis. Veterinary Record, v. 183, n. 11, p. 1-6, 2018.

JANSSENS, L. A. A. Mechanical and Pathophysiological Aspects of Acute Spinal Cord Trauma. Journal of Small Animal Practice, Oxford, v. 32, n. 11, p. 572-578, 1991.

JEFFERY, N. D. Pathophysiology of Spinal Cord Injury. In: Handbook of Small Animal Spinal Surgery. London: Saunders, 1995. p. 64-71.

JEFFERY, N. Neurological Examination of Dogs 1: Techniques. InPractice, v. 23, p. 118-130, 2001a.

JEFFERY, N. Neurological Examination of Dogs 2: Interpretation of Findings. InPractice, v. 23, p. 187-196, $2001 b$.

KERWIN, S. C.; LEVINE, J. M.; HICKS, D. G. Thoracolumbar Spine. In: TOBIAS, K. M.; JOHNSTON, S. A. Veterinary Surgery. Small Animal. Missouri: Elsevier Saunders, v. 1, 2012. p. 449-475.

KLINE, K. L. Terapia Complementar e Alternativa em Pacientes com Doença Neurológica. In: DEWEY C. W.; DA COSTA R. C. Neurologia Canina e Felina: Guia Prático. São Paulo: Guará, 2017. p. 678-687.

LE COUTEUR, R. A.; GRANDY, J. L. Doenças da Medula Espinhal. In: ETTINGER, S. J.; FELDMAN, E. C. Tratado de Medicina Interna Veterinária: Doenças do Cão e do Gato, v. 1. 5 ed. Rio de Janeiro: Guanabara Koogan, 2008. p. 644-694.

LORENZ, M.D.; KORNEGAY, J. N. Handbook of Veterinary Neurology. 4 ed. Saunders, 2004. 468 p.

MACIAS, C.; MCKEE, W. M.; MAY, C.; INNES, J. F. Thoracolumbar Disk Disease in Large Dogs: a Study of 99 Cases. Journal Small Animal Practice, Ontario, v. 43, n. 10, p. 439-446, 2002.

MAI, W. Características das Doenças da Medula Espinhal em Cães e Gatos pela Ressonância Magnética e por Tomografia Computadorizada. In: THRALL D. E. Diagnóstico de Radiologia Veterinária. 6 ed. Philadelphia: Saunders Elsevier, 2014. p. 194-221. 
MCKEE, W. M. A Comparison of Hemilaminectomy (With Concomitant Disc Fenestration) and Dorsal Laminectomy for the Treatment of Thoracolumbar Disc Protrusion in Dogs. Veterinary Record, London, v. 130, n. 14, p. 296-300, 1992.

MOISSONNIER, P.; MEHEUST, P.; CAROZZO, C. Thoracolumbar Lateral Corpectomy for Treatment of Chronic Disc Herniation: Technique Description And Use in 15 Dogs. Veterinary Surgery, Davis, v. 33, n. 6, p. 620-628, 2004.

OLBY, N.; JEFFERY, N. Patogenia das Doenças do Sistema Nervoso Central. In: SLATTER, D. Manual de Cirurgia de Pequenos Animais, v. 1.3 ed. Barueri: Manole, 2007. p. 1132-1147.

PADILHA FILHO, J. G.; SELMI, A. L. Discopatia Cervical no Cão. Tratamento Cirúrgico Através de Fenestração Ventral. Estudo Retrospectivo (1986-1997), Ciência Rural, Santa Maria, v. 29, n. 1, p. 75-78, 1999.

PLATT, S. \& OLBY, N. BSAVA Manual of Canine and Feline Neurology. British Small Animal Veterinary Association. 4 ed. 554 p., 2012.

ROYNARD, P.; FRANK, L.; FOWLER, M. Acupuncture for Small Animal Neurologic Disorders, Veterinary Clinics of North America: Small Animal Practice, v. 48, n. 1, p. 201219, 2018.

RUSSELL S. W.; GRIFFITHS R. C. Recurrence of Cervical Disc Syndrome in Surgically and Conservatively Treated Dogs. Journal of the American Veterinary Medical Association. 153:1412-1417, 1968.

SEIM III, H. B. Princípios Fundamentais de Neurocirurgia. In: FOSSUM, T. W. Cirurgia de Pequenos Animais. 3 ed. Rio de Janeiro: Elsevier, 2008a. p. 1357-1378.

SEIM III, H. B. Cirurgia da Coluna Cervical. In: FOSSUM, T. W. Cirurgia de Pequenos Animais. 3 ed. Rio de Janeiro: Elsevier, 2008b. p. 1402-1459.

SEIM III, H. B. Cirurgia da Coluna Toracolombar. In: FOSSUM, T. W. Cirurgia de Pequenos Animais. 3 ed. Rio de Janeiro: Elsevier, 2008c. p. 1460-1492.

SEIM III, H. B.; WITHROW S. J. Descompressão Ventral para o Tratamento do Disco Intervertebral Cervical Herniado no Cão. In: BOJRAB, M. J. Técnicas atuais em cirurgia de pequenos animais. 3 ed. São Paulo: Roca, 1996. p. 552-556.

SELMI A. L. Discopatias. In: JERICÓ, M. M.; NETO, J. P. A.; KOGIKA, M. M. Tratado de Medicina Interna de Cães e Gatos, v. 2. Rio de Janeiro: Roca, 2015. p. 2145-2147.

SHARP, B. Physiotherapy in Small Animal Practice. InPractice, v. 30, n. 4, p. 190-199, 2008.

SHARP, N. J. H.; WHEELER, S. J. Small Animal Spinal Disorders: Diagnosis and Surgery. 2 ed. Philadelphia: Elsevier Mosby, 2005. 379 p.

SHIMIZU, J.; YAMADA K.; MOCHIDA K.; KATO, T.; MUROYA N.; TERATANI Y.; KISHIMOTO M.; LEE K.; IWASAKI T.; MIYAKE, Y. Comparison of the Diagnosis of Intervertebral Disc Herniation in Dogs by CT Before and After Contrast Enhancement of the Subarachnoid Space, Veterinary Record, v. 165, n. 7, p. 200-202, 2009. 
TAYLOR, S. M. Distúrbios da Medula Espinhal. In: NELSON, R. W.; COUTO, C. G. Medicina Interna de Pequenos Animais. 4 ed. Rio de Janeiro: Elsevier, 2010. p. 1067-1093.

TOOMBS J. P.; WATERS D. J. Afecção do Disco Intervertebral. In: SLATTER, D. Manual de Cirurgia de Pequenos Animais, v. 1. 3 ed. Barueri: Manole, 2007. p. 1193-1208.

TUDURY, E. A.; SEVERO, M. S.; MACIEL, M. J. Frequência de Extrusões de Núcleos Pulposos Cervicais e Toracolombares, em Cadáveres Caninos Submetidos à Técnica de Fenestração, Ciência Rural, Santa Maria, v. 34, n. 4, p. 1113-1118, 2004.

WIDMER, W. R.; THRALL, D. E. Vértebras do Cão e do Gato. In: THRALL D. E. Diagnóstico de Radiologia Veterinária. 6 ed. Philadelphia: Saunders Elsevier, 2014. p. 172-193. 